\title{
Optical Fiber Power Meter Comparison Between NIST and NIM*
}

\section{Vayshenker, D. J. Livigni, X.} Li, and J. H. Lehman

Optoelectronics Division, National Institute of Standards and Technology,

Boulder, CO 80305, USA

\section{J. Li, L. M. Xiong and Z. X. Zhang}

National Institute of Metrology, No. 18, Beisanhuan Donglu Rd., Chaoyang Dist., Beijing 100013, China

igor.vayshenker@boulder.nist.gov david.livigni@boulder.nist.gov xiaoyu.li@boulder.nist.gov john.lehman@boulder.nist.gov jianl@nim.ac.cn

xlmin@nim.ac.cn

zhixinzhang@nim.ac.cn
We describe the results of a comparison of reference standards between the National Institute of Standards and Technology (NIST-USA) and National Institute of Metrology (NIM-China). We report optical fiber-based power measurements at nominal wavelengths of $1310 \mathrm{~nm}$ and $1550 \mathrm{~nm}$. We compare the laboratories' reference standards by means of a commercial optical power meter. Measurement results showed the largest difference of less than 2.6 parts in $10^{3}$, which is within the combined standard $(k=1)$ uncertainty for the laboratories' reference standards.
Key words: international comparison; optical fiber; optical power.
Accepted: July 20, 2010

\section{Introduction}

In our previous work [1-7], we reported the results of international comparisons of reference standards used in the calibration of optical fiber power meters (OFPMs). Those reports describe the results that were obtained by use of open laser beams $[1,4,7]$ and optical fiber cables [2-7] at $1310 \mathrm{~nm}$ and $1550 \mathrm{~nm}$. We also compared internal NIST laser and OFPM standards at

\footnotetext{
* Partial contribution of the National Institute of Standards and Technology; not subject to copyright.
}

several laser wavelengths in the visible and near infrared [8]. In this paper, the reference standards maintained by the two national laboratories (NIST and NIM) were compared at nominal wavelengths of $1310 \mathrm{~nm}$ and $1550 \mathrm{~nm}$ by launching optical power from a reference optical fiber.

For OFPM measurements, the primary standard of both NIST [9] and NIM [10] are cryogenic radiometers that have uncertainties of 2 parts in $10^{4}(k=1)$. Typically, reference standards are calibrated against the primary standards by use of open (free-field) collimated beams, but are generally used with divergent beams of laser light exiting an optical fiber. Most 
primary standards are designed to be used with open beams rather than divergent beams from an optical fiber.

For the comparison of reference standards we used a commercial OFPM, which was calibrated at both national laboratories against their reference standards at nominal wavelengths of $1310 \mathrm{~nm}$ and $1550 \mathrm{~nm}$. This transfer standard is also referred as Device Under Test (DUT). The same reference fiber cable was used by the laboratories, which employed a direct substitution method for their measurements.

\section{NIST and NIM Measurement Systems}

The NIST measurement system, described in detail in [11] and depicted in Fig. 1, consists of fiber-pigtailed laser sources at wavelengths of $1306.5 \mathrm{~nm}$ and $1549.6 \mathrm{~nm}$ (all center wavelengths in this paper are based on refractive index in vacuum), a reference optical fiber cable, and a positioning stage (see doubleheaded arrow) for comparing the NIST reference and transfer standards. The output of each laser source is transmitted through a fiber to a fiber splitter from which about $1 \%$ of the power travels to a monitor detector. The remaining $99 \%$ of the power is transmitted to the reference optical fiber cable that is used in the comparison.

The NIST reference standard is an electrically calibrated pyroelectric radiometer (ECPR) that had been previously calibrated against a primary standard, the NIST Laser Optimized Cryogenic Radiometer (LOCR). The ECPR consists of a thermal detector that is covered with gold black coating. The response of the ECPR does not depend on the wavelength of the incident radiation over the wavelength region of $1300 \mathrm{~nm}-1550 \mathrm{~nm}$ [12].

The NIM measurement system is similar to the NIST system. It consists of fiber-pigtailed laser sources at wavelengths of $1301.2 \mathrm{~nm}$ and $1549.2 \mathrm{~nm}$, reference optical fiber cable, and a positioning stage for comparing the NIM reference and transfer standards. The NIM reference standard, the Electrically Calibrated Absolute Radiometer (ECAR) is a thermal device that had been calibrated against the NIM cryogenic radiometer.

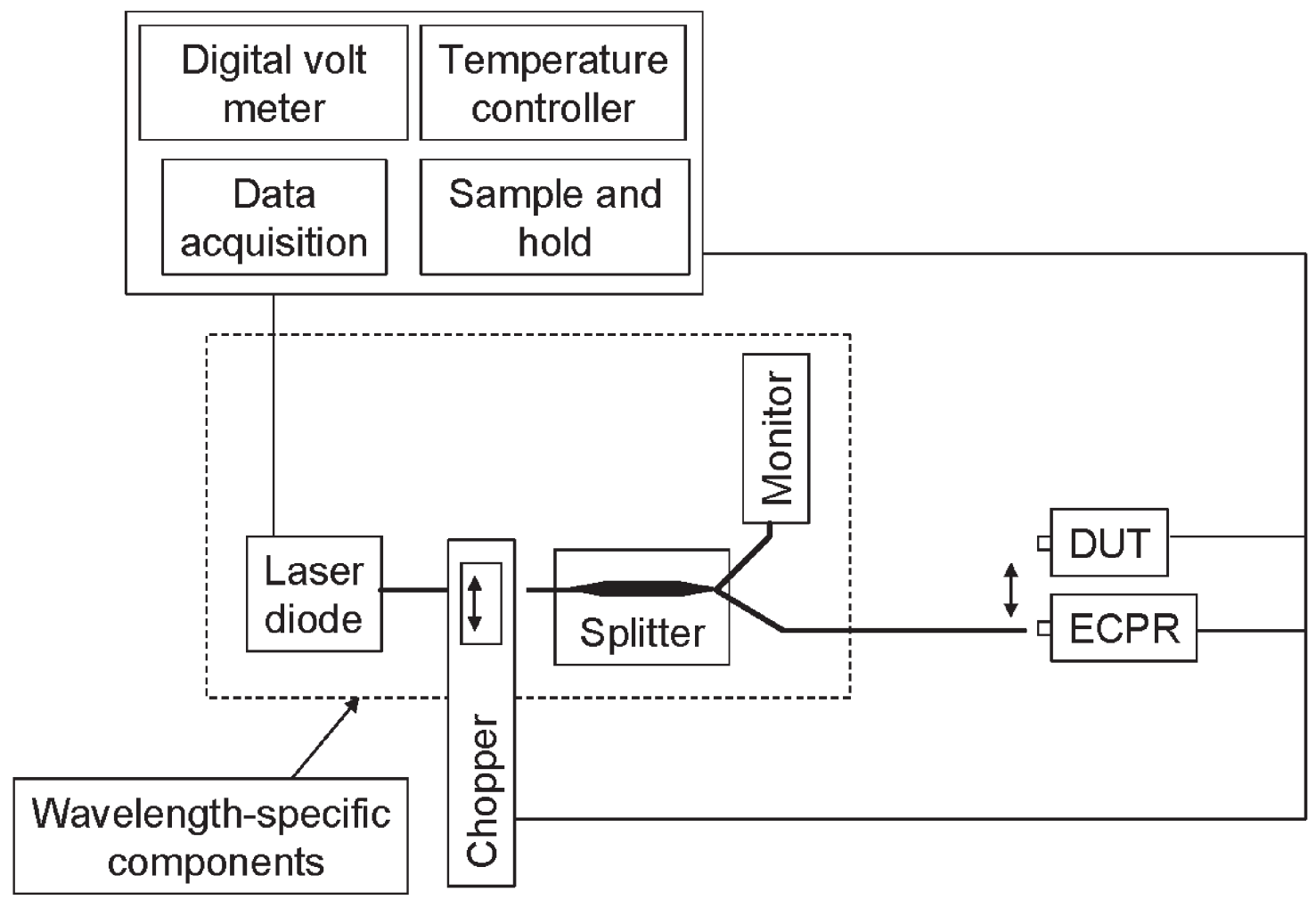

Fig. 1. NIST optical power measurement system. 


\section{Results of the Comparison}

The NIST and NIM reference standards were compared by means of a commercial transfer standard and a reference optical fiber cable at nominal wavelengths of $1310 \mathrm{~nm}$ and $1550 \mathrm{~nm}$. The power was approximately $100 \mu \mathrm{W}(-10 \mathrm{dBm})$. The standard uncertainties for the optical power measurements were evaluated in accordance with ISO document standards [13].

Both laboratories used the same reference optical fiber cable. At NIST eight measurement runs were taken with relative standard deviation of $1 \times 10^{-4}$ at a wavelength of $1310 \mathrm{~nm}$, and six measurement runs were taken with relative standard deviation of $2 \times 10^{-4}$ at a wavelength of $1550 \mathrm{~nm}$. At NIM, nine measurement runs were taken with a relative standard deviation of $0.7 \times 10^{-3}$ at $1310 \mathrm{~nm}$ and a relative standard deviation of $1.5 \times 10^{-3}$ at $1550 \mathrm{~nm}$. The results of the comparison are given in Table 1.

At $1310 \mathrm{~nm}$ the relative difference between the NIST and NIM results was 2.2 parts in $10^{3}$, and at $1550 \mathrm{~nm}$ the relative difference was 2.6 parts in $10^{3}$ (the plus sign for both relative differences indicates that the NIM reference standard read lower than NIST's). The NIST standard uncertainty was 1.9 parts in $10^{3}$ at $1310 \mathrm{~nm}$ and 2.4 parts in $10^{3}$ at $1550 \mathrm{~nm}$, while that of NIM was 3.5 parts in $10^{3}$ at both wavelengths.

Table 1 provides values of relative combined standard uncertainty for NIST and NIM. These values are calculated by taking a square root of the sum of the squares of each laboratory standard uncertainty. The observed interlaboratory differences are less than the relative combined standard $(k=1)$ uncertainties for the laboratories' reference standards.

\section{Conclusion}

This optical power meter comparison shows a good agreement between NIST and NIM measurements. The purpose of this work is to verify a consistency in measurements of optical power in the area of optical telecommunications.

\section{References}

[1] I. Vayshenker, H. Haars, X. Li, J. H. Lehman and D. J. Livigni, Comparison of optical-power meters between NIST and PTB, Metrologia 37, pp. 349-350, 2000.

[2] S. V. Tikhomirov, A. I. Glazov, M. L. Kozatchenko, V. E. Kravtsov, A. B. Svetlichny, I. Vayshenker, T. R. Scott, and D. L. Franzen, Comparison of reference standards for measurements of optical-fibre power, Metrologia 37, 347-348, 2000.

[3] I. Vayshenker, H. Haars, X. Li, J. H. Lehman, and D. J. Livigni, Optical fiber-power meter comparison between NIST and PTB, J. Res. Natl. Inst. Stand. Technol. 108, 391-394, 2003.

[4] I. Vayshenker, J. H. Lehman, D. J. Livigni, X. Li, K. Amemiya, D. Fukuda, S. Mukai, S. Kimura, M. Endo, J. Morel, and A. Gambon, Trilateral optical power meter comparison between NIST, NMIJ/AIST, and METAS, Applied Optics 46 (5), 643-647 (2007).

[5] I. Vayshenker, D. J. Livigni, J. A. Hadler, and J. H. Lehman, NIST optical fiber power measurements: intramural and international comparisons, Digest, Conf. Optical Fibre Measurement Conference, 97-100, Teddington (2007).

[6] I. Vayshenker, J. C. Bermudez, J. C. Molina, Z. E. Ruiz, D. J. Livigni, X. Li, and J. H. Lehman, International comparisons of optical fiber power measurements, Digest, NEWRAD Conf., October, 2008.

[7] I. Vayshenker, J. C. Bermudez, J. C. Molina, Z. E. Ruiz, D. J. Livigni, X. Li, and J. H. Lehman, Bilateral Optical Power Meter Comparison between NIST and CENAM, J. Res. Natl. Inst. Stand. Technol. 113, 4, 1-4, July-August, 2008.

[8] J. H. Lehman, I. Vayshenker, D. J. Livigni, and J. Hadler, Intramural comparison of NIST laser and optical fiber power calibrations, J. Res. Natl. Inst. Stand. Technol. 109, 291-298 (2004).

[9] D. J. Livigni, High accuracy laser power and energy meter calibration service, NIST Special Publication 250-62, 2003.

[10] Y. Lin, L. Lv, and Q. Li, Research on measurement of effective radiant heating power based upon cryogenic radiometer, Acta Metrologica Sinica 30 (6A) (2009).

[11] I. Vayshenker, X. Li, D. J. Livigni, T. R. Scott, and C. L. Cromer, Optical fiber power meter calibrations at NIST, NIST Special Publication 250-54, (2000).

[12] C. A. Hamilton, G. W. Day, and R. J. Phelan Jr., An electrically calibrated pyroelectric radiometer system, Natl. Bur. Stand. (U.S.) Tech. Note 678, March 1976.

[13] ISO, Guide to the Expression of Uncertainty in Measurement, International Organization for Standardization, Geneva, Switzerland, 1993.

Table 1. Results of NIST and NIM comparison

\begin{tabular}{ccccc}
\hline $\begin{array}{c}\text { Source wavelength } \\
(\mathrm{nm})\end{array}$ & $\begin{array}{c}\text { difference } \\
(\%)\end{array}$ & $\begin{array}{c}\text { NIM standard } \\
\text { uncertainty (\%) }\end{array}$ & $\begin{array}{c}\text { NIST standard } \\
\text { uncertainty (\%) }\end{array}$ & $\begin{array}{c}\text { combined standard } \\
\text { uncertainty (\%) }\end{array}$ \\
\hline \multirow{2}{*}{1310} & 0.22 & 0.35 & 0.19 & 0.40 \\
1550 & 0.26 & 0.35 & 0.24 & 0.43 \\
\hline
\end{tabular}


Volume 115, Number 6, November-December 2010

Journal of Research of the National Institute of Standards and Technology

About the authors: Igor Vayshenker, David Livigni, Xiaoyu Li are electronics engineers and calibration leaders, John H. Lehman is a physicist and project leader for Laser Radiometry in the Optoelectronics Division of the NIST Electronics and Electrical Engineering Laboratory of NIST. J. Li, L. M. Xiong and Z. X. Zhang are scientists in the Division of Metrology in Optics and Laser Technology of NIM. The National Institute of Standards and Technology is an agency of the U.S. Department of Commerce. NIM is the National Metrology Institute of China. 